\title{
On the Foundations of Superstring Theory
}

\author{
Gerard't Hooft
}

Received: 16 August 2012 / Accepted: 6 September 2012 / Published online: 22 September 2012

(C) Springer Science+Business Media, LLC 2012

\begin{abstract}
Superstring theory is an extension of conventional quantum field theory that allows for stringlike and branelike material objects besides pointlike particles. The basic foundations on which the theory is built are amazingly shaky, and, equally amazingly, it seems to be this lack of solid foundations to which the theory owes its strength. We emphasize that such a situation is legitimate only in the development phases of a new doctrine. Eventually, a more solidly founded structure must be sought.
\end{abstract}

Although it is advertised as a "candidate theory of quantum gravity", we claim that string theory may not be exactly that. Rather, just like quantum field theory itself, it is a general mathematical framework for a class of theories. Its major flaw could be that it still embraces a Copenhagen view on the relation between quantum mechanics and reality, while any "theory of everything", that is, a theory for the entire cosmos, should do better than that.

Keywords String theory · Black holes · Determinism - QCD · Local conformal symmetry $\cdot$ Hidden variables

The question "what are superstring theory's foundations?" is not just a philosophical one. Just because it is still not understood how to formulate concisely any chain of logical arguments that could reveal what its basic assumptions are, how the theory is constructed part-by-part, what its strengths and limitations are, how many string

G. 't Hooft (ه)

Institute for Theoretical Physics, Utrecht University, Utrecht, The Netherlands

e-mail: g.thooft@uu.nl

url: http://www.phys.uu.nl/ thooft/

G. 't Hooft

Spinoza Institute, Postbox 80.195, 3508 TD Utrecht, The Netherlands

e-mail: foundations.of.physics@ science.uu.nl 
theory scenarios one can imagine, and how, at least in principle, accurate calculations can be performed to decide unambiguously how initial configurations evolve into the future, it is of tantamount importance to carry out as many critical investigations as is possible, to analyze this situation and to reach an agreement that is no longer disputed by a vast majority of the experts. ${ }^{1}$

Yet we see disappointing reluctance in the practitioners of superstring theory to do so. They appear to prefer to discover more and more new "stringy miracles", such as new miraculous matches of black hole microstates, or new cosmological scenarios. If any logical jumps appear to be too large to comprehend, we call these "conjectures", find tests to corroborate the conjectures, and continue our way. These are easier ways to score successes but only deepen and widen the logical depths that block any true understanding.

The situation in standard quantum mechanics, and its extension that incorporates special relativity, now known as quantum field theory, is very different and much more mature. We know what quantum mechanics tells us and what not; we know what quantum field theory can do for us and what it cannot, and why this is so. We do not know this for superstring theory, while any support for the naive expectation that this theory will "solve everything" is rapidly fading.

This short note is not intended to be a critique of superstring theory. The theory has not led to genuine explanations of well-known features of the Standard Model, such as the number of quark and lepton generations, let alone the values of constants such as the finestructure constant or the electron mass, and no definitely testable predictions could be arrived at, but by itself there is nothing wrong with this; such explanations and predictions are still way out of reach for respectable theories of physics. Superstring theory has come closer to potential explanations and predictions than any of its competitors such as loop quantum gravity. Superstring theory does provide for a natural looking framework for the gravitational force acting on fermions, scalar fields and gauge fields, and it does, cautiously, predict an important role for supersymmetry in extensions of the Standard Model that may well be in reach of experiments.

Even if our desire for better foundations of the theory may appear to be a "philosophical" one, it is intended to be much more than that. Our present lack of a deeper, genuine, understanding has not really been an obstacle against progress in the past; physicists are guessing their way around, and they are good at it. Yet it may well form an obstacle against further progress in the future. Conjectures such as the AdS/CFT correspondence appear to be successful, but what do they really mean? Can such conjectures still hold exactly when conformal symmetry in the CFT is explicitly broken? Can they be extended to flat spacetimes? Can the real world be mapped onto its boundary in a meaningful way?

Should a straightforward interpretation of the theory and the prescriptions concerning its application be based on unproven conjectures? Of course we prefer more solid foundations, but on the other hand, the need for unproven conjectures is nothing new or fundamentally rejectable for theories, in particular when they are still in their infancy. The reason for writing this note is simply that the author suspects that

\footnotetext{
${ }^{1}$ String theory has been, and will always be, disputed by numerous onlookers in the sideline who failed to grasp many of its subtle technicalities. It goes without saying that we ignore them.
} 
superstring theory can be improved considerably, or can perhaps be replaced entirely by something better.

What are the examples of better theories? Consider classical mechanics, such as the theory describing the planets in their orbits around the sun, including all mutual disturbances. At first sight, this seems to be a perfect, deterministic, theory, allowing every calculation to be performed with any desired precision, in principle. Of course there are cases where the theory ceases to be valid, such as its description of what happens when two planets collide head-on, or when relativistic or quantum corrections are needed, but this is not the issue. A more subtle objection against the theory of classical mechanics is the inevitable phenomenon of "chaos". What this means is that any error in the initial parameters of a state eventually leads to large deviations in the orbits that are calculated. This implies that the theory is only fully predictive if all masses and initial states are known as infinitely precisely defined real numbers. The need to specify infinite sequences of decimals for all these numbers could be viewed as an unwanted "divergence" of the theory. How can Nature (or "God") continuously remember infinite sequences of decimals? At all stages, the theory requires infinite amounts of information to define how things evolve.

Quantum mechanics usually also works with infinite dimensional Hilbert spaces, which may cause similar difficulties, but here it is easier to imagine how such infinities may be cut-off. Regularization is the removal of states that can rarely be detected or realized in an experiment. Hilbert space is then replaced by a finite dimensional vector space. "Chaos" still takes place, but only in as far as ratios between eigenvalues of the Hamiltonian (more precisely, the separations between eigenvalues) take irrational values. Replacing these by rational values makes our system periodic so that all chaos is removed.

The difficulty with quantum mechanics is that it usually can only give statistical predictions for the outcomes of experiments, which one can also bring forward as an objection: the theory is not infinitely precise in predicting the outcomes of experiments. In practice, this is not a difficulty at all; in any experiments on subatomic particles, we have uncertainties in the initial conditions anyway, so even deterministic theories would give us predictions of a statistical nature and these would be in no way better than the ones provided by standard quantum mechanical calculations. Only when questions are asked about "reality", quantum mechanics fails to give answers of the type sometimes desired.

Quantum field theory is nearly, but just not quite a 'perfect' theory. It is the best possible synthesis of quantum mechanics with special relativity. Apart from the defects of quantum mechanics itself, as just discussed, there are imperfections due to the need to renormalize the interactions, and, associated with that, also the need to consider explicitly perturbation theory. If the theory is chosen to be asymptotically free, only the first few terms of perturbation theory are needed to define the interactions at infinitesimal distance scales; the rest can be calculated, in principle. Rigorous proofs that these calculations always converge have not been given but, considering what we know from perturbation expansions, it is extremely likely that these theories work just fine. If a theory is not asymptotically free, such as the Standard Model itself, calculations will not in general converge but nevertheless suffice to define dozens of decimal places so that there are no difficulties in practice. Of course this does imply 
that such a quantum field theory cannot serve as a model for the ultimate truth; we must continue searching for something better.

A notable feature of all well-established theories of natural phenomena is that they contain 'constants of Nature', some freely adjustable parameters in the form of real numbers. These parameters have to be measured; they cannot be computed from first principles. The Standard Model itself now has some 30 freely adjustable constants.

How does superstring theory compare with these other theories? We are not interested in the fact that string theory was originally introduced to describe the strong interactions. Today, it is claimed to be "the most promising candidate" for a theory that combines general relativity with quantum mechanics, so that it will serve to understand quantum gravity. Considering the successes of quantum field theory in combining special relativity with quantum mechanics, this is evidently an important aim.

Different from quantum field theory, superstring theory hinges on a major, unproven assumption. This is the assertion that most of the particles that were considered to be pointlike in the Standard Model, should be replaced by structures with one internal spacelike dimension: strings. By itself, the assumption is baseless. No experimental evidence can support it. The one and only justification of this assumption is the mathematical observation that, as one of the many constraints required by internal consistency of the resulting scheme, some of the stringlike excitations (the lowest closed string states) behave as gravitons, so that the theory "automatically" generates a gravitational force [1,2]. This surprise was welcomed as a quite pleasing one; apparently, this theory 'generates' gravity. It is the only theory with this bizarre property, and, since gravity undeniably exists, the theory is therefore happily embraced.

An inevitable consequence of this property is mathematical complexity. Gravity is associated to space-time curvature, so, by some miracle, closed string loops turn a flat background spacetime into a dynamical structure, to be provided with a curved coordinate grid. Many other such miracles were encountered. Further self-consistency required the introduction of supersymmetry on the string world sheet, turning it into a super-string, so that string theory also "explains" the existence of fermionic particles $[3,4]$. Next came the observation that higher dimensional membrane like structures also arise as topological features [5]. Probably, what we are arriving at is a fundamental generalization of quantum field theory to include 1-, 2- and higher dimensional subspaces of space-time to replace the elementary particles. One can safely conclude that if we introduce such higher dimensional structures, the emergence of fermions and gravity is inevitable. Yet still, the converse is not obvious; physicists have not succeeded to derive the existence of strings and $D$-branes from the requirements one presumably has to demand for a quantum gravity theory. Therefore, we have to keep in mind the possibility that the real world is something totally different.

This having been said, we can also decide to ignore such objections. We just accept the fact that superstring theory is a theory awaiting further support from experimental evidence. Our job is to provide the proper foundations of the theory.

Consider first the approach starting from the (super)strings themselves. String world sheet diagrams are considered to replace the old Feynman diagrams. Now Feynman diagrams typically represent sequences of perturbative corrections, so that, 
in turn, string world sheets also should be interpreted as perturbative expressions, the perturbation expansion being one in terms of powers of the string coupling constant $g_{s}$. Experiences obtained from the older quantum field theories tell us that such perturbation expansions are fundamentally divergent, generating coefficients of the order of $n$ ! for the $n$th term (the term with $n$ loops in the string world sheet). There is no good reason to expect string perturbation theory to converge better than that. The fact that no $U V$ renormalization appears to be needed might help but certainly does not suffice. In short, string perturbation theory itself definitely does not define a theory. Again looking at quantum field theory, we know of one case where we can do better: in asymptotically free theories such as QCD.

QCD can be defined on a discrete, but arbitrarily dense lattice. The limit $a \downarrow 0$, where $a$ is the size of the meshes, can be rigorously defined, and, according to perturbation expansion, only the first two terms of this expansion need to be known for a rigorous definition of this limit. If any procedure for string theory along similar lines could be defined then we would have more rigorous foundations. Now this is unlikely, since at distance scales tiny compared to the Planck scale, we have no idea about how to formulate what happens, quite unlike asymptotically free field theories. No inductive arguments exist telling us how to integrate the equations starting from a region of triviality such as the short distance region of QCD. Therefore, the situation is exactly as bad as in non-asymptotically free quantum field theories, which are known for their disasters such as the Landau ghosts.

Various cures for this shortcoming have been proposed. It seems that formal mappings may exist of string theories onto infinite-dimensional matrices [6]. Here, however, one also seems to rely on the usefulness of certain $1 / N$ expansions, which again do not converge in general. Are strings finite-dimensional matrices? Those seem to exhibit far too little structure to be able to model a universe as complex as ours, so this would be difficult to accept. If, starting from 10 dimensional superstring theory, we add one more dimension then the string may be seen to be a topological object of a compactified 11 dimensional supergravity. This is an "ordinary" quantum field theory, in so many dimensions that it cannot be asymptotically free. It is sometimes claimed to be finite order by order in perturbation theory, but it seems obvious that perturbation theory itself should be highly divergent here; in any case, this theory also becomes ill-defined at distances small compared to the (11 dimensional version of the) Planck scale.

Further artillery has been put in position. Duality transformations link one kind of (string) theory to others. A problem here is that each of these theories themselves lack solid foundations or definitions. Mappings to and fro won't change that; rigorous foundations are still absent.

In spite of this lamentable situation, miraculous features are claimed by their discoverers, notably in the area of black holes. To demonstrate the inadequacy of (super)string theory, the author has brought forward that black holes show deficiencies that cannot possibly be cured by string theories. Applying standard quantum field theory to black holes exposes a contradiction: it was deduced from quantum field theoretical considerations near a black hole horizon that black holes emit a thermal spectrum of elementary particles. Being thermal, it seems that these particles cannot be in pure quantum states, that is, be described by single vectors in Hilbert space. 
One would have to conclude that black holes themselves cannot obey any Schrödinger equation, since such an equation would require single elements of Hilbert space, even if these are entangled. This contradiction should hold for all local field theories, hence even for string theories.

Now here, we had underestimated string theory. A large class of black holes, that is, the set of black holes that are close to an extreme limit, could be reproduced in string/brane theory, and their internal properties do seem to obey good quantum coherence laws. How can this be?

I do not have the impression that this point is well understood, even by the experts. The horizon of these black holes does not seem to be something one can transform away by coordinate transformations $[7,8]$. What is needed is a theory that explains black hole microstates as a local property of horizons, where the Schwarzschild horizon should be the prototype, not the horizon of an extreme black hole, which is physically different.

Not all hope should be given up. As was shown by the present author, a very special new local symmetry can restore the quantum coherence of black holes: local conformal symmetry [9]. This symmetry must be exact and spontaneously broken. The latter was known and is not at all new; it has been pointed out by many authors; but the claim that this symmetry has to be exact implies that the conformal anomalies have to cancel out, and this is normally not assumed to be the case. We have derived this from ordinary quantum field theory, where it may have deep and important consequences, but the same argument may well hold for string theories as well. Quite possibly, the conformal anomalies cancel out here in a natural way, and this could be a deeper explanation as to why string theories produce quantum mechanically sound black holes. This would indicate that our standard objection has been met; string theory survived it.

One weakness of string theory has not yet been discussed here: the arbitrariness in folding the superfluous dimensions into compact manifolds that may trap arbitrary amounts of different kinds of fluxes. The question how these compactified dimensions came to be folded the way they are seems to be unanswerable: they always were folded this way, from time zero. Not only is this unsatisfactory; it is something of a disaster for the theory, because the compactification ambiguity leads to a permanent large-scale ambiguity in the realization of these theories. There are quadrillions of different theories and there is practically no way to select the one that is appropriate to describe the universe we live in. We find it a curious coincidence that string theory may exhibit so many distinct forms, and that exactly such a set of distinct forms also emerges if we demand the cancellation of conformal anomalies in conventional gravitating quantum field theories.

This might be a signal of an alternative possibility: we conjecture that the "true theory" is something totally different from superstring theory (and certainly also different from gravitating quantum field theories), but that string theory may approximate the truth to various degrees of accuracy in one or several of its compactified realizations, just as it does for some condensed matter systems and QCD.

This conjecture may be exactly as weak as many of the others used in connection with string theory. As long as solid foundations in terms of provable mathematical equations are lacking, one may conjecture anything one likes, it does not help at all 
to strengthen these foundations. Genuine theories should be based on rigorous formulations for their local behavior. Precisely this is a problem: string theory only allows for constructions of on-shell amplitudes, a thing it has in common with predecessors of quantum field theory: axiomatic $S$ matrix theory for hadronic amplitudes. In this theory algebraic symmetries were suspected to suffice, together with dispersion relations, to define dynamical amplitudes, a program that failed bitterly. So, we have to attempt to put our fingers onto local formalisms. This really implies that we have to understand our physical world beyond the Planck scale.

The ultimate theory of the world cannot be a very simple one, if only because it must be able to describe a universe as complex as ours. The hierarchy problem emphasizes that the enormous variety of scales in the universe, both in space and in time, can only be due to the fact that many constants of nature show gigantic variations in strength; the most notable example of this being the value of the cosmological constant in terms of Planck units: close to $10^{-123}$. Since string theories form discrete classes, one has to search for the one representative of these classes that exhibits such a variation in coupling strengths. This might be slightly easier than sometimes claimed. One does not have to dig very deep into mathematics to encounter naturally large numbers such as $e^{e^{2 \pi} / 2}$ or $e^{90 \pi}$, but devising physical theories that naturally produce such quantities might be something of an art.

My last point is one where only few readers will follow me. One of my most fundamental objections against string theory, as usually formulated today, is that it unquestioningly embraces standard quantum theory: states in string theory span a Hilbert space, and its evolution equations are just an arbitrary recipe to generate an evolution operator in this Hilbert space. This implies that string theory also accepts the fact that any given initial state may lead to quantum superpositions of many final states. It accepts that many experiments, even at the Planck level, give rise to outcomes with a probability distribution rather than distinct certainties. This, I believe, cannot be right. The dynamical variables active at the Planck scale should not give rise to quantum vagueness.

This objection only holds for theories that claim to be a theory of everything. Such theories should not be allowed to produce probabilities but only certainties. The most urgent case is the case of a small, compact, evolving universe. In a compact universe, one cannot repeat experiments infinitely many times, and this means that probability theory is inapplicable there. Now, having said this for a compact universe, the same should be true for a non-compact universe if locality of the interactions means anything.

This standpoint clearly calls for the revival of "hidden variables", even "local hidden variables". According to many, the possibility to use such variables was disproved by theorems starting with the Bell inequalities [10]. However, we claim that hidden variables do not exclude being treated as if they occupy quantum states. We can introduce quantum operators even for deterministic hidden variables and end up with quantum models. We explained this in a number of recent articles [11-15], but much earlier a somewhat awkward argument explaining the same was given by D. Bohm [16, 17]. His ideas, involving "pilot wave functions", were dismissed as irrelevant by a majority in the community, even if his claim that he exactly reproduced quantum mechanics was accepted. 
What we showed was that pilot wave functions are not really needed; there are much more elegant and fundamental ways of understanding how quantum mechanics can become emergent $[18,19]$. More recent research lead to a surprise. Attempts to directly reproduce realistic quantum field theories out of deterministic toy models were not totally successful: rotation invariance was difficult to realize, Galilean invariance (needed to describe simple models of moving particles) was even harder, and Lorentz invariance seemed to be hopelessly impossible. The surprise was that the most eminent system ideally suitable to be cast into a deterministic setting, turned out to be string theory. The deterministic equations apply to the world sheet, the dynamical variables are in target space, and there, rotation invariance, even Lorentz invariance, can now be understood [20].

This seems to open up the exciting possibility that various problems can only be solved together at one stroke. It is my fear that without such steps string theory, or any of its more advanced successors, will never be properly understood. Further investigations of string theory's foundations are therefore urgently called for.

\section{References}

1. Friedan, D.H.: Nonlinear models in two + epsilon dimensions. Ann. Phys. 163, 318 (1985)

2. Friedan, D.H.: Nonlinear models in two epsilon dimensions. Phys. Rev. Lett. 45, 1057 (1980)

3. Scherk, J., Schwarz, J.H.: Dual models and the geometry of space-time. Phys. Lett. B 52, 347 (1974)

4. Yoneya, T.: Connection of dual models to electrodynamics and gravidynamics. Prog. Theor. Phys. 51, 1907 (1974)

5. Polchinski, J.: Dirichlet branes and Ramond-Ramond charges. Phys. Rev. Lett. 75, 4724 (1995). hep-th/9510017

6. Banks, T., Fischler, W., Shenker, S.H., Susskind, L.: M theory as a matrix model: a conjecture. Phys. Rev. D 55, 5112 (1997). hep-th/9610043

7. Almheiri, A., Marolf, D., Polchinski, J., Sully, J.: arXiv:1207.3123

8. Susskind, L.: Singularities, firewalls and complementarity. arXiv:1208.3445

9. 't Hooft, G.: The conformal constraint in canonical quantum gravity. arXiv:1011.0061 [gr-qc]

10. Bell, J.S.: On the Einstein-Podolsky-Rosen paradox. Physica 1, 195 (1964)

11. 't Hooft, G.: Quantum mechanics and determinism. hep-th/0105105

12. 't Hooft, G.: How does god play dice? (Pre)determinism at the Planck scale. hep-th/0104219

13. 't Hooft, G.: Determinism in free bosons. Int. J. Theor. Phys. 42, 355 (2003). hep-th/0104080

14. 't Hooft, G.: Determinism and dissipation in quantum gravity. hep-th/0003005

15. 't Hooft, G.: Quantum gravity as a dissipative deterministic system. Class. Quantum Gravity 16, 3263 (1999). gr-qc/9903084

16. Bohm, D.: A suggested interpretation of the quantum theory in terms of 'hidden' variables, I and II. Phys. Rev. 85, 166-193 (1952)

17. Bohm, D.: Proof that probability density approaches $\left.\psi\right|^{2}$ in causal interpretation of quantum theory. Phys. Rev. 89, 458-466 (1953)

18. 't Hooft, G.: Relating the quantum mechanics of discrete systems to standard canonical quantum mechanics .arXiv: 1204.4926

19. 't Hooft, G.: Duality between a deterministic cellular automaton and a bosonic quantum field theory in $1+1$ dimensions. arXiv: 1205.4107

20. 't Hooft, G.: Discreteness and Determinism in Superstrings arXiv:1207.3612 [hep-th] 